\title{
Groups of Fibonacci type revisited
}

\author{
Gerald Williams
}

February 27, 2012

\begin{abstract}
This article concerns a class of groups of Fibonacci type introduced by Johnson and Mawdesley that includes Conway's Fibonacci groups, the Sieradski groups, and the Gilbert-Howie groups. This class of groups provides an interesting focus for developing the theory of cyclically presented groups and, following questions by Bardakov and Vesnin and by Cavicchioli, Hegenbarth, and Repovš, they have enjoyed renewed interest in recent years. We survey results concerning their algebraic properties, such as isomorphisms within the class, the classification of the finite groups, small cancellation properties, abelianizations, asphericity, connections with Labelled Oriented Graph groups, and the semigroups of Fibonacci type. Further, we present a new method of proving the classification of the finite groups that deals with all but three groups.
\end{abstract}

Keywords: Fibonacci group, Fibonacci semigroup, Cyclically presented group, Labelled Oriented Graph (LOG) group, small cancellation theory, asphericity.

MSCs: 20F05, 20F06, 20F10, 57M05, 20F67.

\section{Introduction}

Let $w=w\left(x_{0}, \ldots, x_{n-1}\right)$ be a word in the free group $F_{n}$ with generators $x_{0}, \ldots, x_{n-1}$ and let $\theta: F_{n} \rightarrow$ $F_{n}$ be the automorphism of $F_{n}$ given by $\theta\left(x_{i}\right)=x_{i+1}$ for each $0 \leq i \leq n-1($ subscripts $\bmod n)$. The presentation

$$
\mathcal{G}_{n}(w)=\left\langle x_{0}, \ldots, x_{n-1} \mid w, \theta(w), \ldots, \theta^{n-1}(w)\right\rangle
$$

is said to be a cyclic presentation and the group $G_{n}(w)$ it defines is a cyclically presented group. As part of the programme of research into Fibonacci groups and their generalizations the groups of Fibonacci type $G_{n}(m, k)=G_{n}\left(x_{0} x_{m} x_{k}^{-1}\right)$, defined by the presentations

$$
\mathcal{G}_{n}(m, k)=\left\langle x_{0}, \ldots, x_{n-1} \mid x_{i} x_{i+m}=x_{i+k}(0 \leq i \leq n-1)\right\rangle
$$

$(0 \leq m, k \leq n-1)$ were introduced in [29]. Following the appearance of the groups $G_{n}(m, 1)$ in [21] in connection with Labelled Oriented Graph groups and the independent re-introduction of the groups $G_{n}(m, k)$ in [8] they have enjoyed renewed interest over the last decade [1],[9],[27],[46],[47],[48]. The groups $G_{n}(m, k)$ generalize various groups that have previously been studied: $G_{n}(1,2)$ are Conway's Fibonacci groups $F(2, n)$ of [12], the groups $G_{n}(2,1)$ are the Sieradski groups $S(2, n)$ of [41], and the groups $G_{n}(m, 1)$ are the Gilbert-Howie groups $H(n, m)$ of [21]. (We shall write $\mathcal{F}(2, n), \mathcal{S}(2, n), \mathcal{H}(n, m)$ for the corresponding presentations.) The groups $G_{n}(m, k)$ fit into the wider classes of cyclically presented groups $R(r, n, k, h)$ of [7] and $P(r, n, k, s, q)$ of [38]. The related groups $G_{n}\left(x_{0} x_{m} x_{k}\right)$, introduced in [10], were studied in detail in [19]. 
As well as being of interest in the context of the Fibonacci groups, the groups $G_{n}(m, k)$ provide a hub around which to develop the theory of cyclically presented groups and they have prompted research in combinatorial and computational group theory, semigroup theory, low dimensional topology, algebraic number theory, and linear algebra. In this survey we review current knowledge about their algebraic properties and, in some cases, provide new proofs of known results.

\section{The finite Fibonacci groups and Sieradski groups}

The classification of finite Fibonacci groups $F(2, n)$ is now well known and a survey of the results that led to it is provided in [43].

Theorem 2.1 ([43]) The Fibonacci group $F(2, n)$ is finite if and only if $n=2,3,4,5,7$.

Since the appearance of that survey there have been some further developments that are relevant here. The last of the Fibonacci groups, $F(2,9)$, was first proven to be infinite by Newman [33] using computational algebra: the proof applies a theorem involving a Golod-Shafarevich type inequality to an index 152 subgroup. Holt [24] subsequently used KBMAG to prove that $F(2,9)$ has an automatic structure and that its generators have infinite order. Chalk [11] proved that $F(2, n)$ is infinite for all odd $n \geq 9$ using curvature methods. As well as giving a manual proof that $F(2,9)$ is infinite this filled an important gap in the literature as Lyndon's proof that $F(2, n)$ is infinite for all $n \geq 11$ remains unpublished. There were already alternative proofs that $F(2, n)$ is torsion-free and infinite for all even $n \geq 8$ - see [22],[43]. In connection with this it is worth pointing the following result of Bardakov and Vesnin. (Exercise 12 of [28, page 84] is sometimes, erroneously, cited as a proof.)

Theorem 2.2 ([1, Proposition 3.1]) If $n$ is odd then $F(2, n)$ has non-trivial torsion.

For the Sieradski groups $S(2, n)$ we have the following, which answered a question of Ann-Chi Kim.

Theorem 2.3 ([44]) The group $S(2, n)$ is infinite if and only if $n \geq 6$. It contains a non-abelian free subgroup if and only if $n \geq 7 ; S(2,6)$ is metabelian.

For $n \geq 6$ the proof works by showing that the $\mathbb{Z}_{n}$ extension of $S(2, n)$ has a presentation as a generalized triangle group that has a homomorphism onto an infinite or non-elementary subgroup of $\operatorname{PSL}(2, \mathbb{C})$.

The groups $F(2, n)$ and $S(2, n)$ are also important for geometrical and topological reasons that are described, for example, in [31],[22],[41].

\section{Isomorphisms}

Lemma 3.1 ([1, Lemma 1.2]) The group $G_{n}(m, k)$ is isomorphic to the free product of $(n, m, k)$ copies of $G_{N}(M, K)$ where $N=n /(n, m, k), M=m /(n, m, k), K=k /(n, m, k)$.

Lemma $3.2\left(\left[1\right.\right.$, Lemma 1.1(1,2)] $\quad$ (a) $G_{n}(m, 0)=G_{n}(m, m)=1$;

(b) $G_{n}(0, k)$ is isomorphic to the free product of $(n, k)$ copies of $\mathbb{Z}_{2^{n}-1}$.

By Lemmas 3.1 and 3.2 we may assume (and often will) that $(n, m, k)=1$ and $1 \leq m \neq k \leq n-1$.

Lemma 3.3 ([1, Lemma 1.1(3)]) $G_{n}(m, k) \cong G_{n}(n-m, n-m+k)$. 
By Lemma 3.3 we may also assume $m<k$ (though we will do so less frequently). The following tells us that in certain cases $G_{n}(m, k)$ is isomorphic to a Gilbert-Howie group.

Lemma 3.4 ([1, Lemma 1.3)],[47, Lemma 7]) (a) If $(n, k)=1$ then $G_{n}(m, k) \cong H(n, t)$ where $t k \equiv m \bmod n ;$

(b) if $(n, k-m)=1$ then $G_{n}(m, k) \cong H(n, t)$ where $t(k-m) \equiv n-m \bmod n$.

Similarly we have that if $(n, m)=1$ then $G_{n}(m, k) \cong G_{n}\left(1, k^{\prime}\right)$ where $k^{\prime}=k t$ where $t m \equiv 1 \bmod n$ ([1, Lemma 1.4]). Further isomorphisms are given by the following theorem ([9, Theorem 2]), which is a corrected simplification of [1, Theorem 1.1]. Although developed independently, it is in fact a generalization of $\left[21\right.$, Lemma 2.1] which deals with the case $k=k^{\prime}=1$.

Theorem 3.5 ([1],[9]) Let $G_{n}(m, k)$ and $G_{n}\left(m^{\prime}, k^{\prime}\right)$ satisfy $(n, m, k)=1$ and $\left(n, k^{\prime}\right)=1$. If $m^{\prime}(m-$ $k) \equiv m k^{\prime} \bmod n$ then $G_{n}(m, k) \cong G_{n}\left(m^{\prime}, k^{\prime}\right)$.

For each $n \geq 3$ let $f(n)$ denote the number of pairwise non-isomorphic groups $G_{n}(m, k)$ such that $1 \leq m \neq k \leq n-1$ and $(n, m, k)=1$. Question 2 of [1] asks if $f(n)$ can be computed and this was investigated in [9]. Using the isomorphisms above, computer searches were used to obtain an upper bound $U(n)$ on $f(n)$; invariants of groups (usually the abelianization) were then used to obtain a lower bound $L(n)$. In many cases $U(n)$ and $L(n)$ were found to coincide, therefore giving the value of $f(n)$. For $3 \leq n \leq 27, n \neq 17,19,21,23$ the exact value of $f(n)$ and representatives from each isomorphism class were found [9, Table 1]. In the excluded cases $U(n)$ and $L(n)$ differed by 1 and pairs of possibly isomorphic groups, whose resolution would give the exact value of $f(n)$, were listed. For odd primes $p$ and $l \geq 1$ it was shown that $f(p) \leq(p-1) / 2([9$, Proposition 7$])$ and the values of $f\left(p^{l}\right)$ and $f\left(2^{l}\right)$ were conjectured ([9, Conjecture 8$])$.

\section{The groups with $n \leq 9$}

For $n \leq 6$ all groups $G_{n}(m, k)$ were identified in [29] (see also the tables of [1, Section 4]). In light of Section 3 we assume $(n, m, k)=1,1 \leq m<k \leq n-1$.

Theorem 4.1 ([29]) Let $(n, m, k)=1,1 \leq m<k \leq n-1$. For $n \leq 6$ all the groups $G_{n}(m, k)$ are as follows:

$$
\begin{aligned}
& G_{3}(1,2) \cong Q_{8}, G_{4}(1,2) \cong G_{4}(1,3) \cong \mathbb{Z}_{5}, G_{4}(2,3) \cong S L(2,3), \\
& G_{5}(1,2) \cong G_{5}(1,4) \cong G_{5}(2,3) \cong G_{5}(2,4) \cong \mathbb{Z}_{11}, G_{5}(1,3) \cong G_{5}(3,4) \cong S L(2,5), \\
& G_{6}(1,3) \cong G_{6}(1,4) \cong \mathbb{Z}_{7}, G_{6}(2,3) \cong G_{6}(2,5) \cong \mathbb{Z}_{9}, G_{6}(3,4) \cong G_{6}(3,5) \cong \mathbb{Z}_{2}^{3} \rtimes \mathbb{Z}_{7}, \\
& G_{6}(1,5) \cong G_{6}(1,2)=F(2,6), \text { which is infinite, } \\
& G_{6}(4,5) \cong S(2,6), \text { which is an infinite metabelian group. }
\end{aligned}
$$

(Strictly, in [29] it was established that $G_{6}(2,3)$ is soluble and of order 56; it was identified as $\mathbb{Z}_{2}^{3} \rtimes \mathbb{Z}_{7}$ in [21].) Consider now the case $n=7$. The isomorphisms of Section 3 show that there are three groups: $F(2,7), S(2,7), H(7,3)$ (see also [9, Table 1]). The group $F(2,7) \cong \mathbb{Z}_{29} ; S(2,7)$ contains a free subgroup of rank 2 by Theorem 2.3. Thomas showed that $H(7,3)$ has a normal subgroup of index 256 that is free abelian of rank 8, so is infinite (this is reported in [21]). We summarize this as 
Theorem 4.2 Let $n=7,1 \leq m<k \leq n-1$. Then all the groups $G_{n}(m, k)$ are

$$
\begin{aligned}
& G_{7}(1,2) \cong G_{7}(1,6) \cong G_{7}(2,4) \cong G_{7}(2,5) \cong G_{7}(3,4) \cong G_{7}(3,6) \cong \mathbb{Z}_{29}, \\
& G_{7}(1,3) \cong G_{7}(1,5) \cong G_{7}(2,3) \cong G_{7}(2,6) \cong G_{7}(4,5) \cong G_{7}(4,6) \cong H(7,3), \text { which is infinite, } \\
& G_{7}(1,4) \cong G_{7}(3,5) \cong G_{7}(5,6) \cong S(2,7), \text { which is infinite. }
\end{aligned}
$$

Consider now the case $n=8$. Up to isomorphism there are six groups: $F(2,8), S(2,8), H(8,3)$, $H(8,4), H(8,5), H(8,6)$. The group $F(2,8)$ is infinite by Theorem $2.1 ; S(2,8)$ contains a free subgroup of rank 2 by Theorem 2.3 ; and $H(8,5) \cong \mathbb{Z}_{17}$. As was reported in [1, Section 4] GAP [20] may be used to establish that $G=H(8,3)$ is finite of order $3^{10} \cdot 5=295245$; it may further be used to show that it is soluble of derived length 3 with first, second, and third derived quotients $\mathbb{Z}_{5}, \mathbb{Z}_{3}^{4}, \mathbb{Z}_{3}^{6}$, respectively. This group provides a rare example of a finite, non-metacyclic, cyclically presented group. Other examples are given (for example) in [6]. I am not aware of any cyclic presentation $\mathcal{G}_{n}(w)$ with $n>8$ that defines a finite non-metacyclic group. The groups $H(8,4), H(8,6)$ were proved to be infinite in [21] using the asphericity techniques that we will describe in Section 7 , but we can see this directly by using GAP to show that their second derived quotients are $\mathbb{Z}^{8}$ and $\mathbb{Z}^{12}$, respectively. In summary

Theorem 4.3 Let $n=8,1 \leq m<k \leq n-1,(n, m, k)=1$. Then all the groups $G_{n}(m, k)$ are $F(2,8)=G_{8}(1,2) \cong G_{8}(1,7) \cong G_{8}(3,5) \cong G_{8}(3,6)$, which is infinite, $G_{8}(2,5) \cong G_{8}(6,7) \cong S(2,8)$, which is infinite, $G_{8}(1,3) \cong G_{8}(1,6) \cong G_{8}(5,6) \cong G_{8}(5,7) \cong H(8,3)$, which is finite of order $3^{10} \cdot 5$, $G_{8}(4,5) \cong G_{8}(4,7) \cong H(8,4)$, which is infinite, $G_{8}(1,4) \cong G_{8}(1,5) \cong G_{8}(3,4) \cong G_{8}(3,7) \cong H(8,5) \cong \mathbb{Z}_{17}$, $G_{8}(2,3) \cong G_{8}(2,7) \cong H(8,6)$, which is infinite.

Consider now the groups $G_{n}(m, k)$ for $n=9$. Up to isomorphism there are five groups: $F(2,9)$, $S(2,9), H(9,3), H(9,4), H(9,7)$. By Theorem 2.3 the group $S(2,9)$ contains a free subgroup of rank 2 . The group $F(2,9)$ is infinite (see Theorem 2.1). In [9, Lemma 15] Newman's method of proof ([33]) of that result was successfully applied to (the index 448 second derived subgroup of) the group $H(9,3)$, proving it infinite. This also establishes the previously unknown fact that the group $G(-, 9)$ of $[17$, page 228] is infinite. It is currently unknown if the (non-isomorphic) groups $H(9,4)$ and $H(9,7)$ are infinite.

O'Brien [34] reports that $G=H(9,4)$ has derived quotient $\mathbb{Z}_{19}$, has the following 2-generator 2-relator presentation

$$
\left\langle x, y \mid y^{-2} x^{-2} y^{-1} x^{2} y x y^{-1} x, y^{-1} x^{-1} y^{-2} x^{-1} y^{-1} x y^{-2} x^{-1} y x^{-1}\right\rangle,
$$

and observes that adding the relator $x^{2} y^{-2} x^{2} y x y^{3} x^{-2} y^{-1} x^{-1} y^{-1} x^{-2}$ to this gives $L=P S L(2,8) \times \mathbb{Z}_{19}$. He observes that the kernel $K$ of the epimorphism $G \rightarrow L$ is perfect and is the normal closure of a single element (many of the individual Schreier generators of $K$ are suitable elements). It is not known if $K$ is infinite, finite, or trivial (that is, whether $G \cong L$ ). Little seems to be known about the group $H(9,7)$, other than that its abelianization is $\mathbb{Z}_{37}$. I thank Eamonn O'Brien for allowing me to include this information here.

We summarize the results for $n=9$ in the following theorem. (As there are 27 triples $(n, m, k)$ that satisfy the hypotheses, we no longer list the isomorphism classes.)

Theorem 4.4 Let $n=9,1 \leq m \neq k \leq n-1,(n, m, k)=1$. Then $G_{n}(m, k)$ is isomorphic to exactly one of $F(2,9), S(2,9), H(9,3), H(9,4), H(9,7)$. The groups $F(2,9), S(2,9), H(9,3)$ are infinite; it is unknown whether $H(9,4)$ and $H(9,7)$ are finite or infinite. 


\section{The finite groups}

Question 1 of [1] asks for a classification of the finite groups $G_{n}(m, k)$. For $n \leq 9$ the groups are described in Section 4. For $n \geq 10$ the classification of the finite groups $G_{n}(m, k)$ is given by the following theorem. Note that for $n \geq 10$ the only finite groups are cyclic.

Theorem $5.1([21],[47])$ Let $n \geq 10,1 \leq m \neq k \leq n-1$, and $(n, m, k)=1$. Then $G_{n}(m, k)$ is finite if and only if $2 k \equiv 0 \bmod n$ or $2(k-m) \equiv 0 \bmod n$, in which case $G_{n}(m, k) \cong \mathbb{Z}_{s}$ where $s=2^{n / 2}-(-1)^{m+n / 2}$.

We record

Lemma $5.2([47$, Lemma 3]) Suppose that $(m, k)=1, k \not \equiv 0 \bmod n, k \not \equiv m \bmod n$ and either $2 k \equiv 0 \bmod n$ or $2(k-m) \equiv 0 \bmod n$. Then $G_{n}(m, k) \cong \mathbb{Z}_{s}$ where $s=2^{n / 2}-(-1)^{m+n / 2}$.

(The case $k=1$ was previously obtained in [21, Proposition 2.2].)

The proof of Theorem 5.1 that is currently in the literature combines the asphericity results of [21] and [47] (see Section 7) with the classification of the perfect groups $G_{n}(m, k)$ of [35],[48] (see Section 6) and therefore relies on some involved algebraic number theory. We will present that proof after we have given Theorems 7.4 and 7.5. For the case $n \geq 11$ we now present a new, purely group theoretic, proof that avoids the algebraic number theory of [35],[48]. The automorphism $\theta$ of the introduction induces an action of the cyclic group $T=\left\langle t \mid t^{n}\right\rangle$ of order $n$ on the presentation $G_{n}(m, k)$. Specifically, $t^{-1} x_{i} t=x_{i+1}(0 \leq i \leq n-1)$ and therefore $t^{-i} x_{0} t^{i}=x_{i}$. Writing $x=x_{0}$ we see that the split extension of $G_{n}(m, k)$ by $T$ has a presentation

$$
E_{n}(m, k)=\left\langle x, t \mid t^{n}, x t^{-m} x t^{m-k} x^{-1} t^{k}\right\rangle .
$$

Setting $y=t^{m} x^{-1}$, eliminating $x$, then inverting $t$ gives the alternative presentation

$$
E_{n}(m, k)=\left\langle y, t \mid t^{n}, y^{2} t^{k} y^{-1} t^{m-k}\right\rangle
$$

which is a one-relator product $G=(H * K) /\langle\langle R\rangle\rangle$ where $H=\langle y \mid\rangle, K=\left\langle t \mid t^{n}\right\rangle$, and $R=y^{2} t^{k} y^{-1} t^{m-k}$.

For a one-relator product $G=(H * K) /\langle\langle R\rangle\rangle$ the Freiheitssatz is said to hold if the natural homomorphisms $H \rightarrow G, K \rightarrow G$ are embeddings. The Freiheitssatz for one-relator products has been considered in many papers, for example in [25]. Since $H \cong \mathbb{Z}$, if the Freiheitssatz holds in this setting we have that $E_{n}(m, k)$ (and hence $\left.G_{n}(m, k)\right)$ is infinite. Now $R$ is of the form $R=a b c d$ $(a, c \in H, b, d \in K)$, and the Freiheitssatz for one-relator products where the relator takes this form was studied in [17],[18],[39],[40] and other papers by the same authors. In our proof we will require results from those papers including the following, which we reproduce here as the preprint [39] remains unpublished.

Theorem 5.3 ([39],[40]) Let $G=(H * K) /<<R>>$ be a one-relator product where $R=a^{2} b a^{-1} d$ with $a \in H, b, d \in K, a^{2} \neq 1, b^{2} \neq 1, d^{2} \neq 1, b \neq d^{ \pm 2}, d \neq b^{ \pm 2}$. If $|H| \geq 7,|H| \neq 8,|K| \geq 11$, $K \notin\left\{\mathbb{Z}_{12}, A_{4}, S_{4}, A_{5}\right\}$ then the Freiheitssatz holds.

We now give the new proof of Theorem 5.1 for $n \geq 11$.

\section{Proof of Theorem 5.1 for $n \geq 11$ using Freiheitssatz methods}

Let $a=y, b=t^{k}, d=t^{m-k}$. Then $E_{n}(m, k)=(H * K) /\langle\langle R\rangle\rangle$ where $H=\langle y \mid\rangle, K=\left\langle t \mid t^{n}\right\rangle$, and $R=a^{2} b a^{-1} d$. If $2 k \equiv 0 \bmod n$ or $2(k-m) \equiv 0 \bmod n$ then the condition $(n, m, k)=1$ implies 
$(m, k)=1$ and so the result follows from Lemma 5.2. If $m \equiv 2 k \bmod n$ or $(m+k) \equiv 0 \bmod n$ then $G_{n}(m, k) \cong F(2, n)$ by Lemma 3.3 so the result follows from Theorem 2.1. If $k \equiv 2 m \bmod n$ then $G_{n}(m, k) \cong S(2, n)$ so the result follows from Theorem 2.3. Thus we may assume $2 k \not \equiv 0$, $2(k-m) \not \equiv 0, m \not \equiv 2 k,(m+k) \not \equiv 0, k \not \equiv 2 m$, and by hypothesis we have $k \not \equiv 0, k \not \equiv m, m \not \equiv 0$ (all $\bmod n$ ). Equivalently, in $K$ we have $b^{2} \neq 1, d^{2} \neq 1, b \neq d, d \neq b^{-2}, b \neq d^{-2}, b \neq 1, d \neq 1, b \neq d^{-1}$.

If additionally $(m-k) \not \equiv 2 k \bmod n$ and $k \not \equiv 2(m-k) \bmod n$, or equivalently that (in $K) d \neq b^{2}$, $b \neq d^{2}$ then Theorem 5.3 implies that the Freiheitssatz holds. In particular we have that $H \cong \mathbb{Z}$ embeds in $E_{n}(m, k)$ so it, and hence $G_{n}(m, k)$, is infinite.

Suppose then that $d=b^{2}$ or $b=d^{2}$ in $K$ (equivalently that $(m-k) \equiv 2 k \bmod n$ or $k \equiv$ $2(m-k) \bmod n$, respectively). The condition $(n, m, k)=1$ implies that $(n, k)=1$ when $d=b^{2}$ and $(n, m-k)=1$ when $b=d^{2}$. Thus by applying an automorphism of $\mathbb{Z}_{n}$ we may assume $k=1$ or $(m-k)=1$, respectively. By replacing $y$ and $t$ by their inverses, if necessary, we see that in each case

$$
E_{n}(m, k) \cong\left\langle y, t \mid t^{n}, y^{2} t^{2} y^{-1} t\right\rangle
$$

For each $q \geq 1$ this maps onto

$$
L_{q}=\left\langle y, t \mid y^{q}, t^{n}, y^{2} t^{2} y^{-1} t\right\rangle .
$$

Since $n \geq 11$, Theorem 3 of [18] implies that for any $q \geq 72$, the group $\mathbb{Z}_{q}=\left\langle y \mid y^{q}\right\rangle$ embeds into $L_{q}$. Therefore $\left|E_{n}(m, k)\right| \geq q$ for all $q \geq 72$, and hence is infinite.

Observe that the first half of the proof is also valid for $n=10$, but that the application of [18, Theorem 3] requires $n \geq 11$. Thus if the proof is continued to the case $n=10$ we may assume either $(m-k) \equiv 2 k \bmod n$ or $k \equiv 2(m-k) \bmod n$. The condition $(n, m, k)=1$ means we may assume $k=1$ or $(m-k)=1$ in these cases, respectively, so we are reduced to considering the groups $G_{10}(3,1)=H(10,3)$ and $G_{10}(3,2)$. By Theorem 3.5 these are isomorphic, so there is only one group $G_{n}(m, k)$ with $n \geq 10$ that cannot be proved infinite by this method, namely $H(10,3)$.

\section{Abelianizations}

The abelianization of a finitely presented group is determined by the Smith form of its relation matrix (see for example [28, pages 57-58]). As is described in [28, pages 76-77], in the case of a cyclically presented group $G_{n}(w)$ the relation matrix is a circulant matrix

$$
M_{n}(w)=\operatorname{circ}_{n}\left(a_{0}, \ldots, a_{n-1}\right)=\left(\begin{array}{ccccc}
a_{0} & a_{1} & \ldots & a_{n-2} & a_{n-1} \\
a_{n-1} & a_{0} & \ldots & a_{n-3} & a_{n-2} \\
\vdots & & & \vdots & \\
a_{1} & a_{2} & \ldots & a_{n-1} & a_{0}
\end{array}\right)
$$

where (for $0 \leq i \leq n-1) a_{i}$ is the exponent sum of $x_{i}$ in $w=w\left(x_{0}, \ldots, x_{n-1}\right)$.

The order $\left|G_{n}(w)^{\mathrm{ab}}\right|$ is given by $\left|\operatorname{det}\left(M_{n}(w)\right)\right|$, where 0 is interpreted as $\infty$; in particular $G_{n}(w)^{\mathrm{ab}}$ is infinite if and only $M_{n}(w)$ is singular, and $G_{n}(w)$ is perfect if and only $M_{n}(w)$ is unimodular. Letting

$$
f(t)=\sum_{i=0}^{n-1} a_{i} t^{i}
$$

be the representer polynomial for $M_{n}(w)$ we have the following expression for $\operatorname{det}\left(M_{n}(w)\right)$ as a resul- 
tant:

$$
\begin{aligned}
\operatorname{det}\left(M_{n}(w)\right) & =\operatorname{Res}\left(f(t), t^{n}-1\right) \\
& =\prod_{\theta^{n}=1} f(\theta) .
\end{aligned}
$$

For groups $G_{n}(m, k)$ we have $a_{0}=1, a_{m}=1, a_{k}=-1$ and $a_{i}=0(i \neq 0, m, k)$ so $f(t)$ is the trinomial

$$
f(t)=t^{m}-t^{k}+1
$$

In this case we will write $M_{n}(m, k)=\operatorname{circ}_{n}\left(a_{0}, \ldots, a_{n-1}\right)$.

For the Fibonacci groups $F(2, n)$ the order of $F(2, n)^{\text {ab }}$ is given by the following theorem, where $L_{n}$ denotes the $n$th Lucas number.

Theorem 6.1 (Lyndon, [13]) $\left|F(2, n)^{\mathrm{ab}}\right|=L_{n}-1-(-1)^{n}$.

In particular, $F(2, n)^{\text {ab }}$ is finite for all $n$. Using the theory of elementary divisors, the structure of $F(2, n)^{\mathrm{ab}}$ was determined by Bumby.

Theorem 6.2 (Bumby, [13])

$$
F(2, n)^{\mathrm{ab}}= \begin{cases}\mathbb{Z}_{s} & \text { if }(n, 6)=1, \\ \mathbb{Z}_{2} \oplus \mathbb{Z}_{2 s} & \text { if }(n, 6)=3, \\ \mathbb{Z}_{s} \oplus \mathbb{Z}_{s} & \text { if }(n, 4)=2, \\ \mathbb{Z}_{s} \oplus \mathbb{Z}_{5 s} & \text { if }(n, 4)=4,\end{cases}
$$

(where s can be found from Theorem 6.1).

For the Sieradski groups, the structure of $S(2, n)^{\text {ab }}$ is given by the following theorem, which has been proved using number theory in [30, page 236] and by geometric methods (involving Brieskorn manifolds) in [9, Lemma 9]; additionally the first line was asserted in [41, page 138] and the necessary and sufficient conditions for $S(2, n)^{\text {ab }}$ to be infinite were proved in [44, Theorem A]. Here we give a purely group theoretic proof.

Theorem $6.3([30],[9])$

$$
S(2, n)^{\mathrm{ab}}= \begin{cases}1 & \text { if }(n, 6)=1, \\ \mathbb{Z}_{3} & \text { if }(n, 6)=2, \\ \mathbb{Z}_{2} \oplus \mathbb{Z}_{2} & \text { if }(n, 6)=3, \\ \mathbb{Z} \oplus \mathbb{Z} & \text { if }(n, 6)=6 .\end{cases}
$$

\section{Proof}

In $S(2, n)^{\text {ab }}$ multiplying the relations $x_{i} x_{i+2}=x_{i+1}$ and $x_{i+2} x_{i+4}=x_{i+3}$ gives

$$
x_{i} x_{i+2}^{2} x_{i+4}=x_{i+1} x_{i+3}=x_{i+2}
$$

so $x_{i} x_{i+2} x_{i+4}=1$ and so also $x_{i+2} x_{i+4} x_{i+6}=1$. Together these imply $x_{i}=x_{i+6}$.

Now there exist $\alpha, \beta \in \mathbb{Z}$ such that $6 \alpha+n \beta=(n, 6)$ so $6 \alpha \equiv(n, 6) \bmod n$, and hence

$$
x_{i}=x_{i+6}=x_{i+12}=\ldots=x_{i+6 \alpha}=x_{i+(n, 6)} .
$$


Adding the relation $x_{i}=x_{i+(n, 6)}$ to the presentation of $S(2, n)^{\text {ab }}$ gives

$$
\begin{aligned}
S(2, n)^{\mathrm{ab}} & =\left\langle x_{0}, \ldots, x_{n-1} \mid x_{i} x_{i+2}=x_{i+1}, x_{i}=x_{i+(n, 6)}(0 \leq i \leq n-1)\right\rangle^{\mathrm{ab}} \\
& =\left\langle x_{0}, \ldots, x_{(n, 6)-1} \mid x_{i} x_{i+2}=x_{i+1}(0 \leq i \leq(n, 6)-1)\right\rangle^{\mathrm{ab}} \\
& =S(2,(n, 6))^{\mathrm{ab}}
\end{aligned}
$$

from which the result follows easily.

Question 6 of [1] asks for a formula for $\left|G_{n}(m, k)^{\mathrm{ab}}\right|$. This is a special case of a problem considered by Ore in [36, Section 5], namely to find $\operatorname{det}\left(\operatorname{circ}_{n}\left(a_{0}, \ldots, a_{n-1}\right)\right)$ when exactly 3 of the $a_{i}$ are non-zero (ie when $f(t)$ is a trinomial). Ore asserts that Theorem 1 of [36] may be used to give an explicit statement for the determinant, although the resulting formula (which is not given) is "somewhat complicated". Abelianizations of particular families of groups $G_{n}(m, k)$ have been of interest elsewhere, for example [28, Exercise 11, page 196] concerns the groups $G_{2 s+3}(s+2,2 s+2)^{\text {ab }}$.

Question 6 of [1] further asks if a formula for $\left|G_{n}(m, k)^{\mathrm{ab}}\right|$ could be expressed in terms of numbers generalizing the Fibonacci numbers (as in Theorem 6.1). It is likely that a formula based on numbers defined by recurrence relations exists, but such a formula would be extremely complicated so could not really constitute a positive answer to the last part of this question.

Using the formula (3) necessary and sufficient conditions for $G_{n}(m, k)^{\text {ab }}$ to be infinite can be given. (This generalizes the result for the case $k=1$, which was dealt with in [35, Theorem 2(a)].)

Theorem $6.4\left([35],\left[47\right.\right.$, Theorem 4]) Suppose $(n, m, k)=1$. Then $G_{n}(m, k)^{\text {ab }}$ is infinite if and only if $n \equiv 0 \bmod 6$ and $((m \bmod 6, k \bmod 6)=(2,1)$ or $(4,5))$.

Based on experiments using GAP the necessary and sufficient conditions for $G_{n}(m, k)$ to be perfect were conjectured in [47] and proved in [48].

Theorem $6.5([48])$ Suppose $(n, m, k)=1$. Then $G_{n}(m, k)$ is perfect if and only if $((n, 6)=1$ and $m \equiv 2 k \bmod n)$ or $k \equiv 0 \bmod n$ or $k \equiv m \bmod n$.

Since $G_{n}(k, k)=1$ and $G_{n}(m, 0)=1$ it is clear that $m \equiv k \bmod n$ and $k \equiv 0 \bmod n$ are sufficient conditions. Since the conditions $(n, m, k)=1,(n, 6)=1$ and $m \equiv 2 k$ imply that $G_{n}(m, k) \cong S(2, n)$, the fact that these are also sufficient conditions follows from Theorem 6.3. (This was also proved in $[47$, Lemma 5].) That these conditions are necessary is more difficult, and this was the main result of [48]. In number theoretic terms, letting $f(t)=t^{m}-t^{k}+1$, where $m, k \in \mathbb{Z}, k \neq 0, m \bmod n$, and $(n, m, k)=1$, we can state this as:

(i) Suppose $(n, 6)=1$. If $\operatorname{Res}\left(f(t), t^{n}-1\right)= \pm 1$ then $m \equiv 2 k \bmod n$.

(ii) Suppose $(n, 6)>1$. Then $\operatorname{Res}\left(f(t), t^{n}-1\right) \neq \pm 1$.

The proof of (i) is essentially a re-run of the proof for the case $k=1$ ([35, Lemma 3.1]), and was given in [48, Lemma 4.8]. The proof of (ii) for the case $k=1$ from [35] does not directly generalize, but a proof (inspired by the methods of [35]) was provided in [48]. We shall not present that proof here; rather we prove a weaker form, namely that (ii) can be false for at most finitely many $n$. (We include this proof as it is rather different to the proof for all $n$, and it may be of use for future research in this area.)

Theorem 6.6 Let $f(t)=t^{m}-t^{k}+1$, where $m, k \in \mathbb{Z}, k \neq 0, m \bmod n$. Then there are at most finitely many $n$ with $(n, m, k)=1$ and $(n, 6)>1$ such that $\operatorname{Res}\left(f(t), t^{n}-1\right)= \pm 1$. 


\section{Proof}

If $f$ is reducible then the main result of [45] implies that $f$ has a root of modulus 1 . A technical proposition then shows that $f$ must have a factor $g(t)=t^{2(m, k)}-t^{(m, k)}+1$ so $f(t)=f_{1}(t) g(t)$ for some $f_{1}(t)$. Since $(n, 6)>1$ Theorem 6.3 implies $R_{n}(g) \neq \pm 1$ and because $R_{n}\left(f_{1}\right) \in \mathbb{Z}$ we have that

$$
\operatorname{Res}\left(f(t), t^{n}-1\right)=R_{n}\left(f_{1}\right) R_{n}(g) \neq \pm 1 .
$$

Thus we may assume that $f$ is irreducible.

Suppose further that $f$ is cyclotomic, $\Phi_{L}$, say. If $L=2^{s}$ (for some $s$ ) $\Phi_{L}(t)=t^{2^{s-1}}+1 \neq f(t)$, so we may assume $L$ is not a power of 2 . Therefore, by [15, Theorem 1] the middle coefficient of $\Phi_{L}(t)$, and hence of $f(t)$, is odd. Now (for $k \neq m$ ) $f(t)$ only has an odd middle coefficient if $k=2 m$ or $m=2 k$ in which case $\operatorname{Res}\left(f(t), t^{n}-1\right) \neq \pm 1$ by Theorems 6.1 and 6.3 , respectively.

Thus we may assume that $f$ is irreducible and not cyclotomic. Since $k \neq m$ we have (by replacing $f(t)$ by $f\left(t^{-1}\right)$, if necessary) that $f$ is of positive degree and is not equal to $\pm t$. Then Theorem 1 of [35] (or Proposition 1 of [14]) implies that there are at most finitely many $n$ for which $\operatorname{Res}\left(f(t), t^{n}-1\right)= \pm 1$.

\section{Asphericity}

Associated to a group presentation is the standard 2-complex which has a single 0-cell, 1-cells in oneto-one correspondence with the generators, and 2-cells in one-to-one correspondence with the relators. We will not distinguish between the presentations and the 2-complexes, and we will talk of homotopy equivalences (denoted by the symbol $\simeq$ ) and homotopy groups of presentations. Further, we will say that a presentation $\mathcal{P}$ is (topologically) aspherical if the second homotopy group $\pi_{2}(\mathcal{P})=0$. Here we describe results which give an almost complete classification of the aspherical presentations $\mathcal{G}_{n}(m, k)$.

The proof of Lemma 3.1 partitions the generators and relations and relabels them and in fact proves

Lemma 7.1 The the presentation $\mathcal{G}_{n}(m, k)$ is the disjoint union of $(n, m, k)$ presentations, each of which is homotopy equivalent to the presentation $\mathcal{G}_{N}(M, K)$ where $N=n /(n, m, k), M=m /(n, m, k)$, $K=k /(n, m, k)$.

Clearly the presentations $\mathcal{G}_{n}(m, 0)$ and $\mathcal{G}_{n}(m, m)$ are aspherical. A group defined by an aspherical presentation with no proper powered relators is torsion-free, so presentations defining non-trivial groups are not aspherical. In particular, Lemma 3.2 implies

Corollary $7.2 \mathcal{G}_{n}(0, k)$ is not aspherical.

The upshot of this is that we may again assume that $(n, m, k)=1$ and $1 \leq m \neq k \leq n-1$.

The proof of Lemma 3.4 works by relabelling generators, so it is really a result about homotopy equivalence of presentations rather than isomorphisms. We record this as

Lemma $7.3 \quad$ (a) If $(n, k)=1$ then $\mathcal{G}_{n}(m, k) \simeq \mathcal{H}(n, t)$ where $t k \equiv m \bmod n$;

(b) if $(n, k-m)=1$ then $\mathcal{G}_{n}(m, k) \simeq \mathcal{H}(n, t)$ where $t(k-m) \equiv n-m \bmod n$.

Therefore, if $(n, k)=1$ or $(n, k-m)=1$ then $\mathcal{G}_{n}(m, k) \simeq \mathcal{H}\left(n, m^{\prime}\right)$ for some $m^{\prime}$. For this reason we can divide results into a statement of the classification of the aspherical Gilbert-Howie presentations $\mathcal{H}(n, m)$ with $m \neq 0,1$ and a statement of the aspherical presentations $\mathcal{G}_{n}(m, k)$ with $(n, m, k)=1$, $1 \leq m \neq k \leq n-1,(n, k)>1,(n, k-m)>1$. For the first category we have 
Theorem 7.4 ([21, Theorem 3.2]) Suppose $n \geq 3,2 \leq m \leq n-1$ and assume $(n, m) \neq(9,4),(9,7)$. Then $\mathcal{H}(n, m)$ is aspherical unless $(n, m)$ is one of the following, in which case it is not aspherical:

$(n, 2)(n \geq 3),(n, n-1)(n \geq 3),(2 t-1, t)(t \geq 3),(2 t-2, t)(t \geq 3),(6,3),(7,3),(7,5),(8,3),(9,3),(9,6)$.

In the same way as it remains unknown whether the (non-isomorphic) groups $H(9,4), H(9,7)$ are finite or infinite, it is likewise unknown whether or not the presentations $\mathcal{H}(9,4), \mathcal{H}(9,7)$ are aspherical. When the paper [21] was written, the status of $\mathcal{H}(8,3)$ was unknown; since we now know that $H(8,3)$ is finite and non-trivial we can conclude that $\mathcal{H}(8,3)$ is not aspherical. Similarly, the fact that $\mathcal{H}(6,3)$ and $\mathcal{H}(2 t-2, t)(t \geq 3)$ are not aspherical follows from the fact that the groups they define are finite and non-trivial. It is possible to explicitly draw homotopy spheres for the presentations $\mathcal{H}(n, 2)=\mathcal{S}(2, n)$ and $\mathcal{H}(n, n-1) \simeq \mathcal{F}(2, n)$ and $\mathcal{H}(2 t-1, t) \simeq \mathcal{F}(2,2 t-1)$ (see for example [31]), so these presentations are not aspherical. In the remaining two cases $\mathcal{H}(7,3) \simeq \mathcal{H}(7,5)$ and $\mathcal{H}(9,3) \simeq \mathcal{H}(9,6)$ it is again possible to draw spheres explicitly to show that they are not aspherical. As these are not provided in [21] we give them in Figures 1 and 2.

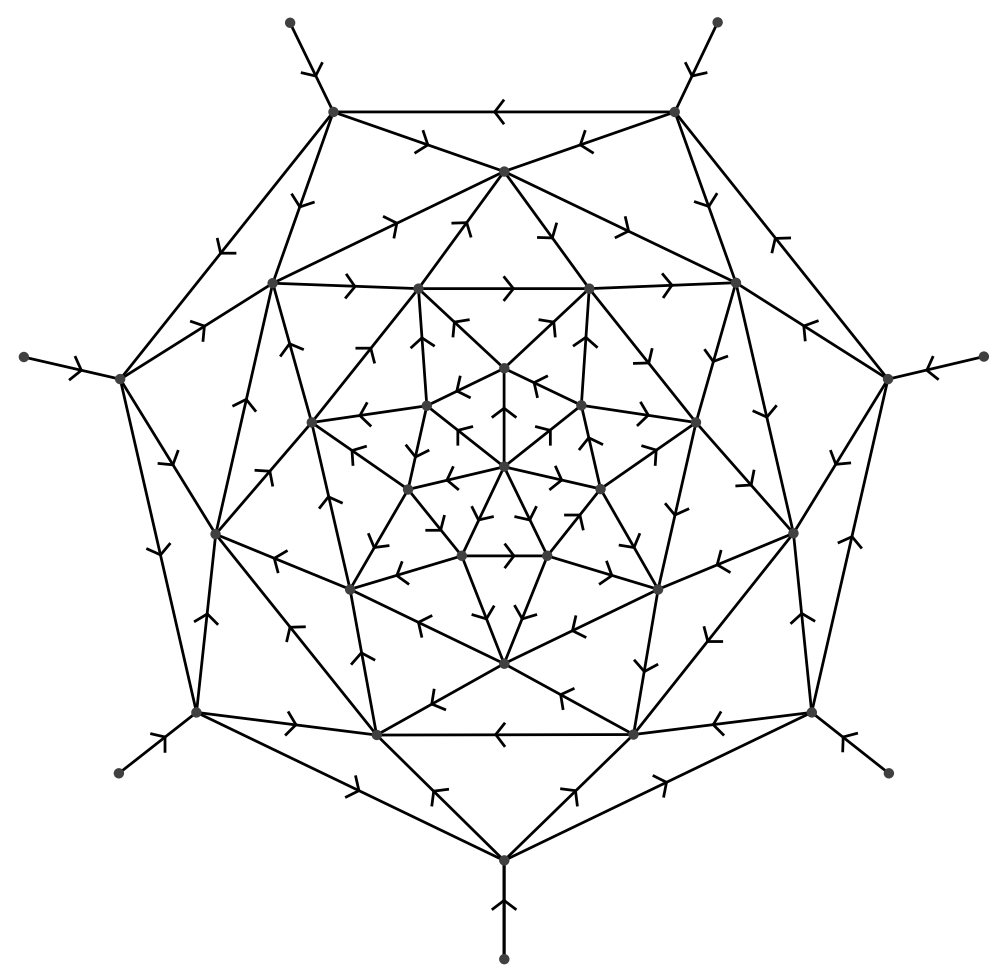

Figure 1: Homotopy sphere over $\mathcal{H}(7,3)$.

For the remaining presentations $\mathcal{G}_{n}(m, k)$ we have the following result [47, Theorem 2], which builds on [1, Theorem 2.1].

Theorem 7.5 ([47, Theorem 2]) Suppose $n \geq 2,1 \leq m \neq k \leq n-1,(n, m, k)=1,(n, k)>1$, $(n, k-m)>1$. Then $\mathcal{G}_{n}(m, k)$ is aspherical unless $(m, k)=1$ and either $2 k \equiv 0 \bmod n$ or $2(k-m) \equiv$ $0 \bmod n$, in which case $\mathcal{G}_{n}(m, k)$ is not aspherical.

The fact that $\mathcal{G}_{n}(m, k)$ is not aspherical in the indicated cases of Theorem 7.5 is due to the fact that the corresponding groups are finite and non-trivial (by Lemma 5.2). The proofs of Theorems 7.4 and 7.5 use the same underlying technique, which we now outline. The $\mathbb{Z}_{n}$-extension $E_{n}(m, k)$ of $G_{n}(m, k)$ given in (1) has a relative presentation

$$
\mathcal{R}_{n}(m, k)=\left\langle x, T \mid x t^{-m} x t^{m-k} x^{-1} t^{k}\right\rangle
$$




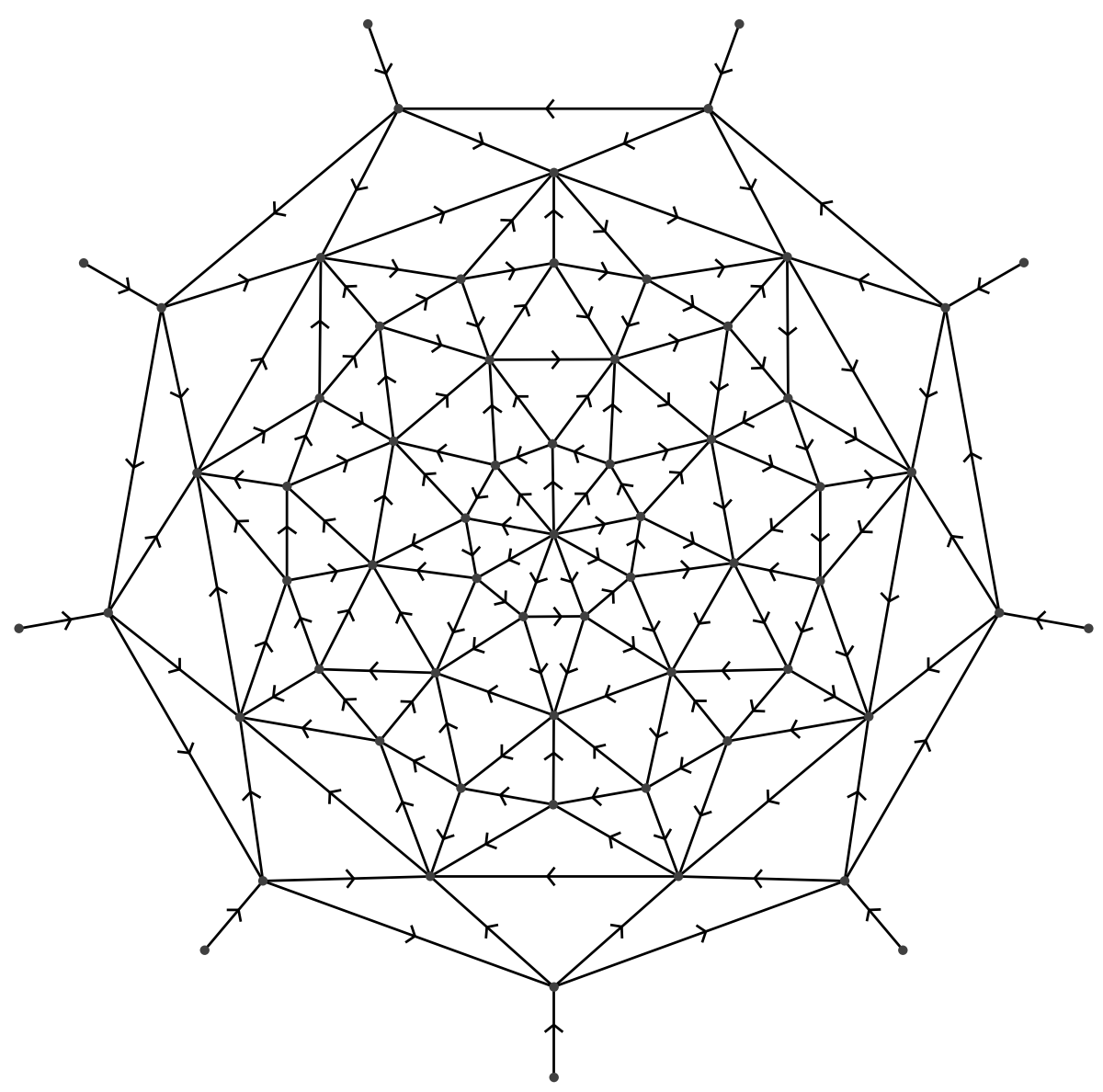

Figure 2: Homotopy sphere over $\mathcal{H}(9,3)$.

where $T=\left\langle t \mid t^{n}\right\rangle$. (For background on relative presentations see [3].) The following crucial lemma was proved by Gilbert and Howie in the case $k=1$; Bardakov and Vesnin performed the routine generalization to the general case.

Lemma 7.6 ([21, Lemma 3.1],[1, Lemma 2.2]) If $\mathcal{R}_{n}(m, k)$ is relatively aspherical then the $a b$ solute presentation $\mathcal{G}_{n}(m, k)$ is topologically aspherical.

Relative presentations of the form $\left\langle x, T \mid x a x b x^{-1} c\right\rangle$ (where $b, c$ are non-trivial elements of $T$ of finite order) had been considered by Edjvet in [16] where, subject to a few exceptions, the classification of the relatively aspherical presentations was given. Applying this to $\mathcal{R}_{n}(m, k)$ gives conditions for $\mathcal{G}_{n}(m, k)$ to be aspherical. (For the cases with $(n, m, k)=1,(n, k)>1$ and $(n, k-m)>1$ this was done in [1, Theorem 2.1]). The arguments presented above show that $\mathcal{G}_{n}(m, k)$ is not aspherical in all remaining cases.

We can now give the proof of Theorem 5.1 that was mentioned in Section 5 .

\section{Proof of Theorem 5.1 using asphericity and classification of the perfect groups}

If $2 k \equiv 0 \bmod n$ or $2(k-m) \equiv 0 \bmod n$ then the condition $(n, m, k)=1 \operatorname{implies}(m, k)=1$ and so the result follows from Lemma 5.2. If $m \equiv 2 k \bmod n$ then $(n, m, k)=1 \operatorname{implies}(n, k)=1$ so $G_{n}(m, k) \cong S(2, n)$ by Lemma 3.4 , which is infinite by Theorem 2.3. Thus we may assume $m \not \equiv 2 k$, $2 k \not \equiv 0,2(k-m) \not \equiv 0($ all $\bmod n)$. 
By Theorem 6.5 the group $G_{n}(m, k)$ is not perfect, so it is non-trivial. If $(n, k)>1$ and $(n, k-m)>$ 1 then Theorem 7.5 implies that $\mathcal{G}_{n}(m, k)$ is aspherical, so $G_{n}(m, k)$ is infinite. Thus we may assume that $(n, k)=1$ or $(n, k-m)=1$, so $G_{n}(m, k)$ is isomorphic to $H\left(n, m^{\prime}\right)$ for some $m^{\prime}$ (by Lemma 3.4). Under our derived conditions Theorem 7.4 implies that $\mathcal{H}\left(n, m^{\prime}\right)$ is aspherical, and therefore infinite, unless $\left(n, m^{\prime}\right)=(n, n-1)$ or $(2 t-1, t)$, in which case $H\left(n, m^{\prime}\right) \cong F(2, n)$ (by Lemma 3.3 and Theorem 3.5), which is infinite by Theorem 2.1 .

\section{Small cancellation groups}

In this section we consider small cancellation conditions for the presentations $\mathcal{G}_{n}(m, k)$. We refer the reader to [32, Chapter V] for basic definitions regarding small cancellation theory. Using the characterization (from [23]) of the $\mathrm{C}(3)-\mathrm{T}(q)$ conditions in terms of the star graph, the $\mathrm{C}(3)-\mathrm{T}(6)$ and $\mathrm{C}(3)-\mathrm{T}(7)$ presentations $\mathcal{G}_{n}(m, k)$ were classified in [27]. (The term special means that all relators have length 3 - as is the case for $\mathcal{G}_{n}(m, k)$ - and that the star graph is isomorphic to the incidence graph of a finite projective plane.)

Theorem 8.1 ([27]) Let $A=k, B=m-k$.

(a) The presentation $\mathcal{G}_{n}(m, k)$ is a $C(3)$ - $T(6)$ presentation if and only if $t A \not \equiv 0, t B \not \equiv 0(1 \leq t \leq 5)$ and $A \not \equiv \pm B, A \not \equiv \pm 2 B, B \not \equiv \pm 2 A$ (all mod $n$ ), in which case it is a non-special $C(3)-T(6)$ presentation.

(b) The presentation $\mathcal{G}_{n}(m, k)$ is a $C(3)-T(7)$ presentation if and only if $t A \not \equiv 0, t B \not \equiv 0(1 \leq t \leq 6)$ and $A \not \equiv \pm 2 B, A \not \equiv \pm 3 B, B \not \equiv \pm 2 A, B \not \equiv \pm 3 A, 2 A \not \equiv \pm 2 B$ (all $\bmod n$ ).

The $\mathrm{C}(3)-\mathrm{T}(6)$ presentations $\mathcal{G}_{n}\left(x_{0} x_{m} x_{k}\right)$ were classified in [19, Lemma 5.1]. Extending that proof to classify the special $\mathrm{C}(3)-\mathrm{T}(6)$ presentations it turns out that (up to homotopy equivalence) there is precisely one, namely $\mathcal{G}_{7}\left(x_{0} x_{1} x_{3}\right)$, which was identified as being a special $\mathrm{C}(3)-\mathrm{T}(6)$ presentation in [26, Example 6.3]. Combining these observations with Theorem 8.1 we can now conclude that (up to homotopy equivalence) it is the only special $\mathrm{C}(3)-\mathrm{T}(6)$ cyclic presentation.

In Corollary 8.2 we observe some consequences, most of which are well known properties of $\mathrm{C}(3)$ $\mathrm{T}(6)$ and $\mathrm{C}(3)-\mathrm{T}(7)$ groups (for details see the references in [27]). A countable group $G$ is said to be $S Q$-universal if every countable group can be embedded in a quotient group of $G$; in particular, SQ-universal groups contain a free subgroup of rank 2 . The dependence problems [37] $\mathrm{DP}(n), n \geq 1$, generalize the word problem (DP(1)) and the conjugacy problem $(\mathrm{DP}(2))$. A group is Hopfian if it is not isomorphic to any of its proper quotients.

Corollary $8.2([27]) \quad$ (a) Suppose the conditions of Theorem 8.1(a) hold. Then $G_{n}(m, k)$ has solvable word and conjugacy problems and is automatic, $S Q$-universal, torsion-free, and acts properly and cocompactly on a $C A T(0)$ space.

(b) Suppose the conditions of Theorem 8.1(b) hold. Then $G_{n}(m, k)$ has solvable dependence problems $D P(n)$ for all $n \geq 1$ and is hyperbolic, Hopfian, and acts properly and cocompactly on a CAT(-1) space, and the class of groups $G_{n}(m, k)$ has solvable isomorphism problem.

Using Corollary 8.2 and [9, Proposition 7] it can be shown (see [27]) that for odd primes $p, G_{p}(m, k)$ is either the trivial group, the Fibonacci group $F(2, p)$, or the Sieradski group $S(2, p)$ or is isomorphic to $G_{p}(1,(p+1) / 3)($ when $p \equiv 2 \bmod 3)$ or to $G_{p}(1,(p+2) / 3)($ when $p \equiv 1 \bmod 3)$, or $\mathcal{G}_{p}(m, k)$ is 
$\mathrm{C}(3)-\mathrm{T}(7)$. A group $G_{n}(m, k)$ may be hyperbolic and contain a free subgroup of rank 2 without its presentation $\mathcal{G}_{n}(m, k)$ satisfying the $\mathrm{C}(3)-\mathrm{T}(6)$ or $\mathrm{C}(3)$ - $\mathrm{T}(7)$ conditions; for example $H(n, 3)$ is such a group for all $n \geq 11, n \neq 12,14([27])$.

\section{$9 \quad$ LOG groups}

A Labelled Oriented Graph ( $L O G$ ) consists of a finite connected graph (possibly with loops and multiple edges) with vertex set $V$ and edge set $E$ together with three maps $\iota, \tau, \lambda: E \rightarrow V$ called the initial vertex map, terminal vertex map, and labelling map, respectively. The LOG determines a corresponding LOG presentation

$$
\left\langle V \mid \tau(e)^{-1} \lambda(e)^{-1} \iota(e) \lambda(e)(e \in E)\right\rangle
$$

a group with a LOG presentation is a $L O G$ group.

Following [42] we call the semidirect product

$$
\widehat{G}_{n}(w)=G_{n}(w) \rtimes_{\phi} \mathbb{Z}=G_{n}(w) *_{\phi}=\left\{G_{n}(w), t \mid t^{-1} g t=\phi(g), g \in G_{n}(w)\right\}
$$

the natural $H N N$ extension of $G_{n}(w)$. The connection between the HNN extension of a cyclically presented group and LOG groups was investigated in [21],[42]. For the groups $G_{n}(m, k)$ we have

Theorem 9.1 ([27]) The natural HNN extension $\widehat{G}_{n}(m, k)$ of $G_{n}(m, k)$ is the LOG group corresponding to the $L O G$ in Figure 3.
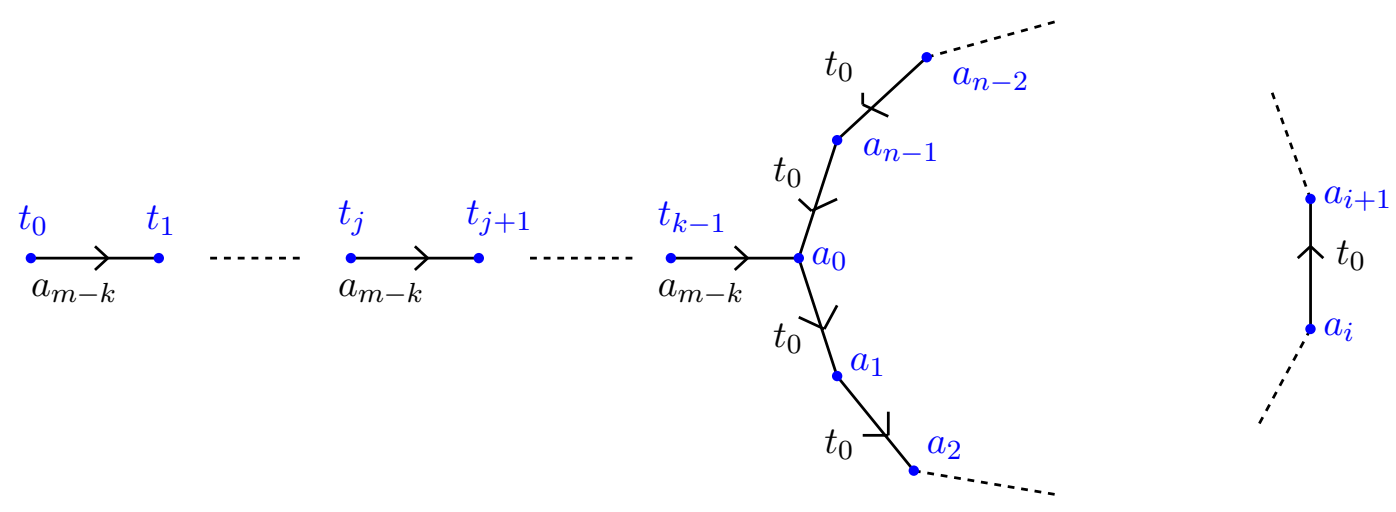

Figure 3: LOG corresponding to $\widehat{G}_{n}(m, k)$.

Restricting to $k=1$ gives the LOG in [21] for the natural HNN extension $\widehat{H}(n, m)$ of $H(n, m)$; restricting to $k=1$ and $n=2$ gives the LOG in [2, Section 4] which, as a LOG group with torsion, was the initial motivation for the investigations in [21].

\section{Semigroups}

Each presentation $\mathcal{G}_{n}(m, k)$ is a positive presentation, so may be regarded as a semigroup presentation; we let $S_{n}(m, k)$ denote the semigroup it defines. As Ruškuc points out in his thesis, Wicks' solution ([13]) to Conway's question [12] makes no use of inverses and hence proves that the semigroup given by the generators and relations of $F(2,5)$ is cyclic of index 1 and period 5 , and so the study of Fibonacci semigroups is as old as the study of Fibonacci groups. The following theorem is a special case of [46, Theorem B] (a generalization of [4, Theorem 1.2] combined with [5, Theorem 3.5]), which 
deals with the semigroups and groups defined by the 4-parameter cyclic presentations $\mathcal{R}(r, n, k, h)$ of $[7]$.

Theorem 10.1 ([46]) The semigroup $S_{n}(m, k)$ is finite if and only if $((n, k)=1$ or $(n, m-k)=1)$ and $G_{n}(m, k)$ is finite, in which case $S_{n}(m, k)$ is respectively the union of $(n, m-k)$ disjoint left ideals or $(n, k)$ disjoint right ideals, each isomorphic to $G_{n}(m, k)$.

Since, with the exception of the groups $H(9,4)=G_{9}(4,1)$ and $H(9,7)=G_{9}(7,1)$ the finite groups $G_{n}(m, k)$ have been classified, Theorem 10.1 classifies (up to isomorphism and anti-isomorphism) the finite semigroups $S_{n}(m, k)$. The semigroups $S_{9}(4,1)$ and $S_{9}(7,1)$ are the disjoint unions of 3 copies of $G_{9}(4,1)$ and $G_{9}(7,1)$, respectively, so completing the classification of the finite semigroups $S_{n}(m, k)$ is equivalent to completing the classification of the finite groups $G_{n}(m, k)$.

\section{Acknowledgements}

I would like to thank the following people for enlightening discussions about the various aspects of this work: Bill Bogley, Colin Campbell, Alberto Cavicchioli, Martin Edjvet, Peter Higgins, Jim Howie, John Merriman, Eamonn O’Brien, Nik Ruskuč, Robert Shwartz, Agnese Telloni.

\section{References}

[1] V.G. Bardakov and A.Yu. Vesnin. A generalization of Fibonacci groups. Alg. and Logic, 42:131160, 2003.

[2] W.A. Bogley. Local collapses for diagrammatic reducibility. In Topology and Combinatorial Group Theory, Lect. Notes Math. 1440, pages 27-38, 1990.

[3] W.A. Bogley and Stephen J. Pride. Aspherical relative presentations. Proc. Edin. Math. Soc., 35(1):1-39, 1992.

[4] C.M. Campbell, E.F. Robertson, N.Ruškuc, and R.M. Thomas. Fibonacci semigroups. J. Pure Appl. Algebra, 94(1):49-57, 1994.

[5] C.M. Campbell, E.F. Robertson, N.Ruškuc, and R.M. Thomas. Rewriting a semigroup presentation. Int. J. Algebra Comput., 5(1):81-103, 1995.

[6] C.M. Campbell. Computation techniques and the structure of groups in a certain class. In Proceedings of the 1976 ACM Symposium on Symbolic and Algebraic Computation, pages 312321. ACM Press, New York, 1976.

[7] C.M. Campbell and E.F. Robertson. A note on Fibonacci type groups. Can. Math. Bull., 18:173$175,1975$.

[8] Alberto Cavicchioli, Friedrich Hegenbarth, and Dušan Repovš. On manifold spines and cyclic presentations of groups. In Knot theory, volume 42, pages 49-56. Warszawa: Polish Academy of Sciences, Institute of Mathematics, Banach Cent. Publ., 1998.

[9] Alberto Cavicchioli, E.A. O'Brien, and Fulvia Spaggiari. On some questions about a family of cyclically presented groups. J. Algebra, 320(11):4063-4072, 2008. 
[10] Alberto Cavicchioli, Dušan Repovš, and Fulvia Spaggiari. Families of group presentations related to topology. J. Algebra, 286(1):41-56, 2005.

[11] Christopher P. Chalk. Fibonacci groups with aspherical presentations. Comm. Algebra, 26(5):1511-1546, 1998.

[12] J. Conway. Advanced problem 5327. Am. Math. Mon., 72(8):915, 1965.

[13] J. Conway et. al. Solution to advanced problem 5327. Am. Math. Mon., 74(1):91-93, 1967.

[14] J.E. Cremona. Unimodular integer circulants. Math. Comp., 77:1639-1652, 2008.

[15] Gregory P. Dresden. On the middle coefficient of a cyclotomic polynomial. Am. Math. Mon., 111(6):531-533, 2004.

[16] Martin Edjvet. On the asphericity of one-relator relative presentations. Proc. R. Soc. Edinb., Sect. A, 124(4):713-728, 1994.

[17] Martin Edjvet and Arye Juhász. Equations of length 4 and one-relator products. Math. Proc. Camb. Philos. Soc., 129(2):217-229, 2000.

[18] Martin Edjvet and Arye Juhász. One-relator quotients of free products of cyclic groups. Commun. Algebra, 28(2):883-902, 2000.

[19] Martin Edjvet and Gerald Williams. The cyclically presented groups with relators $x_{i} x_{i+k} x_{i+l}$. Groups, Geom., Dyn., 4(4):759-775, 2010.

[20] The GAP Group. GAP - Groups, Algorithms, and Programming, version 4.4.9, 2006.

[21] N.D. Gilbert and James Howie. LOG groups and cyclically presented groups. J. Algebra, 174(1):118-131, 1995.

[22] H. Helling, A.C. Kim, and J.L. Mennicke. A geometric study of Fibonacci groups. J. Lie Theory, 8(1):1-23, 1998.

[23] Patricia Hill, Stephen J. Pride, and Alfred D. Vella. On the T $(q)$-conditions of small cancellation theory. Isr. J. Math., 52:293-304, 1985.

[24] Derek F. Holt. An alternative proof that the Fibonacci group $F(2,9)$ is infinite. Exp. Math., 4(2):97-100, 1995.

[25] James Howie. How to generalize one-relator group theory. Combinatorial group theory and topology, Sel. Pap. Conf., Alta/Utah 1984, Ann. Math. Stud. 111, 1987.

[26] James Howie. On the SQ-universality of T(6)-groups. Forum Math., 1(3):251-272, 1989.

[27] James Howie and Gerald Williams. Tadpole Labelled Oriented Graph groups and cyclically presented groups. Preprint.

[28] D.L. Johnson. Topics in the Theory of Group Presentations, volume 42 of London Mathematical Society Lecture Note Series. Cambridge University Press, 1980.

[29] D.L. Johnson and H. Mawdesley. Some groups of Fibonacci type. J. Aust. Math. Soc., 20:199-204, 1975. 
[30] D.L. Johnson and R.W.K. Odoni. Some results on symmetrically-presented groups. Proc. Edinb. Math. Soc., II. Ser., 37(2):227-237, 1994.

[31] A.C. Kim. On the Fibonacci group and related topics. L.A.Bokut (ed.) et al., Second international conference on algebra dedicated to the memory of A. I. Shirshov. Proceedings of the conference on algebra, August 20-25, 1991, Barnaul, Russia. Providence, RI: American Mathematical Society. Contemp. Math. 184, 231-235, 1995.

[32] Roger C. Lyndon and Paul E. Schupp. Combinatorial group theory, volume 89 of Ergebnisse der Mathematik und ihrer Grenzgebiete. Springer-Verlag, 1977.

[33] M.F. Newman. Proving a group infinite. Arch. Math., 54(3):209-211, 1990.

[34] E.A. O’Brien. Personal communication, 13/08/2009.

[35] R.W.K. Odoni. Some Diophantine problems arising from the theory of cyclically-presented groups. Glasg. Math. J., 41(2):157-165, 1999.

[36] Oystein Ore. Some studies on cyclic determinants. Duke Math. J., 18:343-354, 1951.

[37] Stephen J. Pride. Star-complexes, and the dependence problems for hyperbolic complexes. Glasg. Math. J., 30(2):155-170, 1988.

[38] M.I. Prishchepov. Asphericity, atoricity and symmetrically presented groups. Comm. Algebra, 23(13):5095-5117, 1995.

[39] Robert Shwartz. On the Freiheitssatz in certain one-relator free products. II. Preprint.

[40] Robert Shwartz. On the Freiheitssatz in certain one-relator free products with a single relator of length 4. PhD thesis, Technion - Israel Institute of Technology, 1999.

[41] Allan J. Sieradski. Combinatorial squashings, 3-manifolds, and the third homology of groups. Invent. Math., 84:121-139, 1986.

[42] A. Szczepański and A. Vesnin. HNN extension of cyclically presented groups. J. Knot Theory Ram., 10(8):1269-1279, 2001.

[43] Richard M. Thomas. The Fibonacci groups revisited. Groups St. Andrews 1989, Vol. 2, Proc. Int. Conf., St. Andrews 1989, Lond. Math. Soc. Lect. Note Ser. 160, 445-454, 1991.

[44] Richard M. Thomas. On a question of Kim concerning certain group presentations. Bull. Korean Math. Soc., 28(2):219-224, 1991.

[45] H. Tverberg. On the irreducibility of the trinomials $x^{n} \pm x^{m} \pm 1$. Math. Scand., 8:121-126, 1960.

[46] Gerald Williams. Fibonacci type semigroups. Alg. Coll. to appear.

[47] Gerald Williams. The aspherical Cavicchioli-Hegenbarth-Repovš generalized Fibonacci groups. J. Group Theory, 12(1):139-149, 2009.

[48] Gerald Williams. Unimodular integer circulants associated with trinomials. Int. J. Number Theory, 6(4):869-876, 2010. 